\title{
Columnar/Lamellar packing in cocrystals of arylbipyridines with diiodoperfluorobenzene
}

Remya Ramakrishnan ${ }^{1}$, Ajith R Mallia ${ }^{1}$, Niyas M A ${ }^{1}$, Ramarani Sethy ${ }^{2}$, Mahesh Hariharan ${ }^{1}$

${ }^{1}$ School Of Chemistry, IISER-Thiruvananthapuram, Thiruvanathapuram, India, ${ }^{2}$ Graduate school of Material Science, Nara Institute of Science and Technology (NAIST), Ikoma, Japan

E-mail: remyaramakrishnan16@iisertvm.ac.in

Crystal engineering of polyaromatic hydrocarbons to yield 1-2D lamellar arrangement is vital for photonic and semiconductor applications. Construction of supramolecular self-assembled solid state architectures with desirable packing motifs has gained tremendous attention in terms of its potential applications in various crystal engineering fields. Highly directional intermolecular interactions such as hydrogen bonding, dihydrogen and halogen bonding play pivotal role in driving the formation of supramolecular scaffolds for the design of functional materials. Cocrystallization is one such bottom up approach in understanding intermolecular interactions and producing aggregated supramolecular crystal structures. Our continued efforts in modulating the packing arrangement in near-orthogonal bichromophores [1] and PAH's [2] encouraged us to engineer halogen bonded cocrystals and explore the close packing. Crystalline arylated 2,2'-bipyridines and corresponding cocrystals with the coformer 1,4-diiodotetrafluorobenzene were successfully synthesized and the nature of intermolecular interactions were closely investigated by hirshfeld surface (HS), quantum theory of atoms in molecules

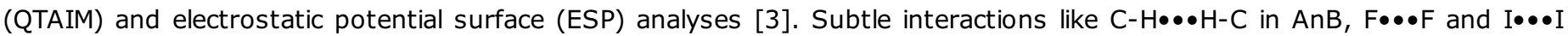
(van der Waals') observed in $(\mathrm{NaB})_{2} \bullet \mathrm{D}_{2.5}$ were characterized by QTAIM-IQA method. The studies indicate that the strongly directional and energetically favorable $\mathrm{C}-\mathrm{I} \bullet \bullet \mathrm{N}$ interactions direct formation of co-ordination networks in most of the cocrystals while the $\mathrm{C}-\mathrm{H} \bullet \bullet \mathrm{N}$ hydrogen bonding controls the close packing in ArB derivatives. The hydrogen bonded chains linked by $\mathrm{C}-\mathrm{H} \bullet \bullet \mathrm{F}$ interactions contribute to the two-dimensional packing in cocrystals. Three-dimensional packing arrangement undergoes a transition from sandwich herringbone and herringbone in ArB to lamellar ( $\gamma$-motif) and columnar

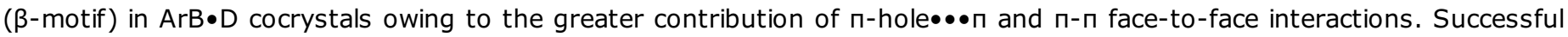
implementation of $\mathrm{C}$ - $\mathrm{I} \bullet \bullet \mathrm{N}$ interactions aided by $2,2^{\prime}$-bipyridine and $\mathrm{D}$ enabled the tuning of three dimensional close packing in planar polyaromatic hydrocarbons into columnar/lamellar arrangement suitable for optoelectronic devices.

[1] Mallia, A. R. et al. (2015). J. Am. Chem. Soc. 137, 15604-15607

[2] Rajagopal, S. K. et al. (2014). Chem. Commun. 50, 8644-8647.

[3] Ramakrishnan, R. et al. (2016). Cryst. Growth Des. 16, 6327-6336.

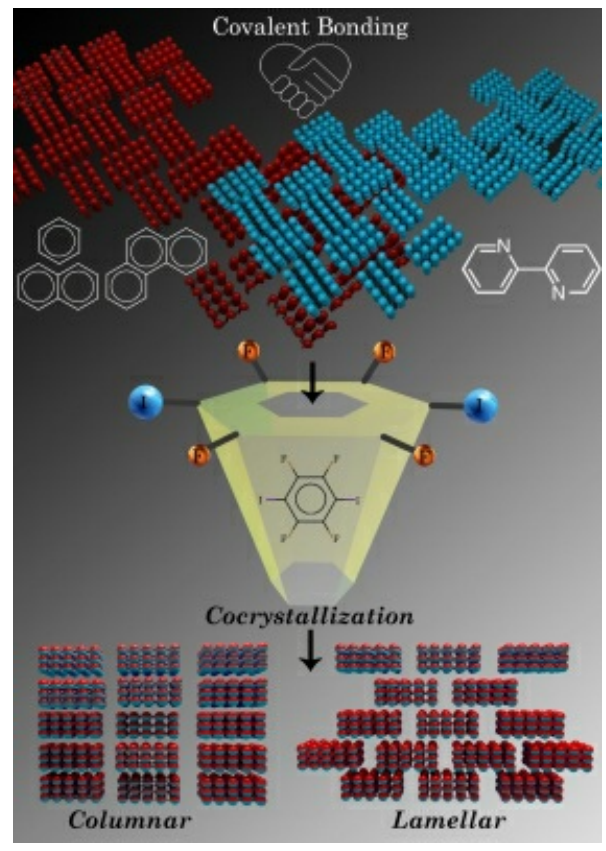

Keywords: 1-2D lamellar arrangement, hirshfeld surface (HS), quantum theory of atoms in molecules (QTAIM) and electrostatic potential surface (ESP) analyses 